\title{
Sexual orientation and suicidal behaviour in young people ${ }^{\dagger}$
}

\author{
Nick Meader and Melissa K. Y. Chan
}

\section{Summary}

Lesbian, gay, and bisexual (LGB) young people have been found to be at greater risk of suicidal behaviour. National prevention strategies have identified the need to reduce suicide risk in this population. However, research on specific risk factors for LGB young people that might inform suicide prevention programmes are at an early stage of development.

\author{
Declaration of interest \\ None.
}

\section{Copyright and usage}

(c) The Royal College of Psychiatrists 2017
Nick Meader (pictured) is a research fellow at the Centre for Reviews and Dissemination at the University of York. Melissa K. Y. Chan is affiliated with the Department of Social Work and Social Administration at the University of Hong Kong.

\section{Background}

Lesbian, gay, bisexual and transgender (LGBT) young people are thought to be at greater risk of suicidal behaviour than heterosexual young people. For example, a recent UK study of LGBT young people found $58 \%$ planned or attempted suicide. ${ }^{1}$ LGB young people report $78 \%$ greater odds of experiencing a common mental disorder compared with heterosexuals ${ }^{2}$ and are more likely to engage in risk behaviours (such as risky sexual behaviour, smoking, illegal drug use). ${ }^{3}$

Queer Futures, a large recent UK study of LGBT young people, identified a number of factors that had an impact on suicidal feelings including experiencing discrimination on the basis of sexual orientation or gender identity; societal norms about gender and sexuality that made them feel ashamed; feeling unable to talk about their distress, self-harm, sexual orientation or gender identity. In addition, there were a variety of other factors that were unrelated to sexual orientation or gender identity such as physical or mental health problems, financial difficulties and bereavement. ${ }^{1}$ Similarly, Youth Chances, another recent large UK study, found high levels of discrimination experienced by LGBT young people and greater risk of experiencing mental health problems. ${ }^{4}$

Proponents of the minority stress model argue that both individual (such as experiencing personal acts of prejudice, stigma and discrimination) and social stressors (such as being a member of a stigmatised minority group which may lead to expectations of discrimination and internalisation of societal attitudes) contribute to increased risk of suicidal behaviour in LGBT groups. ${ }^{5}$ These stressors interact with general risk factors for suicidal behaviour resulting in greater risk for LGBT groups. Other general models of suicidal behaviour that have been applied specifically to LGBT populations include the interpersonal-psychological theory of suicide where burdensomeness and thwarted belongingness are posited as key mechanisms. ${ }^{6}$

The greater risk experienced by LGBT young people is recognised in prevention policies internationally. For example, the National

${ }^{\dagger}$ See pp. 77-87, this issue.
Suicide Prevention Strategy for England recommends a targeted approach to reducing risk of suicide in this population. ${ }^{7}$ However, substantial improvements to services are needed to provide effective support and intervention. Many LGBT young people report a reluctance to seek help that partly stems from their experiences as a stigmatised minority. Few areas in the UK provide services that specifically address the needs of LGBT young people. Those who accessed primary care or mental health services were frequently dissatisfied with their care. Healthcare professionals and other staff were often reported to have limited knowledge and understanding of LGBT issues. ${ }^{1,4}$ The Royal College of Nursing and Public Health England have developed a toolkit for nurses to aid them in preventing suicide in LGBT youth. ${ }^{8}$

\section{Meta-analysis of sexual orientation and suicidal behaviour in young people}

A meta-analysis in 2011 found that sexual orientation was associated with greater suicidality. ${ }^{9}$ Miranda-Mendizábal et al, ${ }^{10}$ in this issue, provide a more updated (i.e. including more recent studies) and refined analysis of the literature (for example, with a greater focus on longitudinal studies, separating suicide attempts and suicide as outcomes, investigating risk factors for LGB young people). Based on eight cohort studies, LGB sexual orientation was associated with twofold increased odds (odds ratio $(\mathrm{OR})=2.32$, 95\% CI 1.59-3.39) of a suicide attempt compared with heterosexual orientation. Data on completed suicide were inconclusive. Most studies were judged to have appropriately taken into account confounding. However, each of the included studies used different covariates in their adjusted analyses, therefore this may have contributed to heterogeneity in the meta-analysis. Length of follow-up was adequate in just over half of included studies. Classification of sexual orientation differed widely across studies. The reported mean age ranged from 15 to 19 years. Data on risk factors for LGB young people were limited; the only possible meta-analysis was for depression, which suggested depression may not be a risk factor for suicide attempts in LGB young people $(\mathrm{OR}=1.05,95 \%$ CI $0.93-1.19){ }^{10}$

\section{Future research}

This meta-analysis is a helpful summary of the current state of knowledge on risk of suicidal behaviour in LGB youth. These findings confirm earlier meta-analyses that LGB young people 
are at greater risk of suicidal behaviour than heterosexual young people. Furthermore, they confirm the need for preventive strategies aiming to reduce the risk for LGB youth engaging in suicidal behaviour.

However, a limitation of the review is that it does not look at suicidal behaviour in transgender young people who may have greater risk of suicidality because of the higher rates of stigma. In addition, it was not possible to assess differences in risk between lesbian, gay and bisexual young people. For example, it is unclear if support for bisexual young people within mental health services may differ from that received by other LGBT groups since bisexuality is sometimes not perceived as a fixed sexual identity in the way other sexual orientations are. As a result they may not receive as much support as other groups, which could lead to further marginalisation.

In terms of future research needs, Miranda-Mendizábal et al clarify that studies mainly reported suicide attempts with only one study reporting data on completed suicide. ${ }^{10}$ Therefore further research is needed to assess the association between sexual orientation and completed suicide in young people. There is also a dearth of research on risk factors associated with suicidal behaviour in LGBT youth. For example, it is important to explore whether the risk factor profile for suicidal behaviour in the general population differs from that of LGBT youth.

Evidence is still limited on protective factors and preventive strategies to reduce risk of suicidal behaviour in LGBT youth. There is an emerging evidence base that suggests a positive school environment where there is support available for LGBT students (such as gay-straight alliance groups, policies prohibiting homophobia) is associated with positive outcomes such as reduced suicidal ideation and suicide attempts. ${ }^{11}$

Nick Meader, MSC, PhD, Centre for Reviews and Dissemination, University of York York, UK; Melissa K. Y. Chan, MSc, University of Hong Kong, Hong Kong

Correspondence: Nick Meader, Centre for Reviews and Dissemination University of York, York Y010 5DD, UK. Email: nick.meader@york.ac.uk

First received 31 Jan 2017, final revision 30 May 2017, accepted 5 June 2017

\section{References}

1 McDermott E, Hughes E, Rawlings V. Queer Futures: Understanding Lesbian, Gay, Bisexual and Trans (LGBT) Adolescents' Suicide, Self-Harm, and Help-Seeking Behaviour. Lancaster University, 2016 (http:// www.queerfutures.co.uk/wp-content/uploads/2016/06/Queer-Futures-FinalReport.pdf).

2 Semlyen J, King M, Varney J, Hagger-Johnson G. Sexual orientation and symptoms of common mental disorder or low wellbeing: combined metaanalysis of 12 UK population health surveys. BMC Psychiatry 2016; 16: 67.

3 Centers for Disease Control and Prevention. Sexual identity, sex of sexual contacts, and health-related behaviors among students in grades 9-12United States and selected sites, 2015. MMWR Surveill Summ 2016; 65: $1-202$.

4 Metro Charity. Youth Chances: Integrated Report. Metro Charity, 2016 (https://www.metrocentreonline.org/pdfs/Experiences_LGBTQ_Intergrated \%20Report2015.pdf)

5 Meyer I. Prejudice, social stress, and mental health in lesbian, gay, and bisexual populations: conceptual issues and research evidence. Psychol Bull 2003; 129: 674-97.

6 Joiner T, Pettit JW, Walker RL, Voelz ZR, Cruz J, Rudd MD, et al. Perceived burdensomeness and suicidality: two studies on the suicide notes of those attempting and those completing suicide. J Soc Clin Psychol 2002; 21: $531-45$.

7 Department of Health. Preventing Suicide in England: A Cross-Governmental Outcomes Strategy to Save Lives. Department of Health, 2012.

8 Public Health England \& Royal College of Nursing. Preventing Suicide among Lesbian, Gay and Bisexual Young People: A Toolkit for Nurses. Public Health England, 2015

9 Marshal M, Dietz L, Friedman M. Suicidality and depression disparities between sexual minority and heterosexual youth: a meta-analytic review. Adolesc Health 2011; 49: 115-23.

10 Miranda-Mendizábal A, Castellví P, Parés-Badell O, Almenara J, Alonso I, Blasco MJ, et al. Sexual orientation and suicidal behaviour in adolescents and young adults: systematic review and meta-analysis. Br J Psychiatry 2017; 211: $77-87$.

11 Saewcy EM, Konishi C, Rose HA, Homma Y. School-based strategies to reduce suicidal ideation, suicide attempts, and discrimination among sexual minority and heterosexual adolescents in Western Canada. Int $J$ Child Youth Family Stud 2014; 1: 89-112. 